\title{
Assessing low-light cameras with photon transfer curve method
}

\author{
Luchang $\mathrm{Li}^{*, \dagger}$, Mengting $\mathrm{Li}^{*, \dagger}$, Zhaoning Zhang*,† \\ and Zhen-Li Huang*,,+ \\ *Britton Chance Center for Biomedical Photonics \\ Wuhan National Laboratory for Optoelectronics-Huazhong \\ University of Science and Technology \\ Wuhan 430074, P. R. China \\ ${ }^{\dagger}$ Key Laboratory of Biomedical Photonics of Ministry of Education \\ Department of Biomedical Engineering, Huazhong \\ University of Science and Technology \\ Wuhan 430074, P. R. China \\ tleo@mail.hust.edu.cn
}

Received 14 March 2016

Accepted 18 April 2016

Published 19 May 2016

\begin{abstract}
Low-light camera is an indispensable component in various fluorescence microscopy techniques. However, choosing an appropriate low-light camera for a specific technique (for example, single molecule imaging) is always time-consuming and sometimes confusing, especially after the commercialization of a new type of camera called sCMOS camera, which is now receiving heavy demands and high praise from both academic and industrial users. In this tutorial, we try to provide a guide on how to fully access the performance of low-light cameras using a well-developed method called photon transfer curve (PTC). We first present a brief explanation on the key parameters for characterizing low-light cameras, then explain the experimental procedures on how to measure PTC. We also show the application of the PTC method in experimentally quantifying the performance of two representative low-light cameras. Finally, we extend the PTC method to provide offset map, read noise map, and gain map of individual pixels inside a camera.
\end{abstract}

Keywords: Photon transfer curve; EMCCD; sCMOS; SNR.

\$Corresponding author.

This is an Open Access article published by World Scientific Publishing Company. It is distributed under the terms of the Creative Commons Attribution 4.0 (CC-BY) License. Further distribution of this work is permitted, provided the original work is properly cited. 


\section{Introduction to Low-Light Cameras}

\subsection{A brief history to the development of low-light cameras}

Low-light detection is a hot topic in contemporary research community. Different from conventional detectors, low-light camera is a special kind of lowlight detector which is designed to image weak signal comparable to the camera's electronic noise (called camera noise). ${ }^{1}$ Low-light cameras have been used intensively for detecting single molecule fluorescence. ${ }^{2,3}$ There are typically four types of widely-used low-light cameras, including cooled charge-coupled device (CCCD), electron multiplier CCD (EMCCD), intensified CCD (ICCD) and scientific complementary metal oxide semiconductor (sCMOS) cameras. ${ }^{1}$ Besides, other kinds of low-light cameras such as electron-multiplying complementary metal oxide semiconductor (CMOS) camera and electron-bombarded CCD cameras are being developed and tested in research labs. ${ }^{4,5}$

CCD consists of an array of light-sensitive pixels (photodiodes) that can convert photons to electrons and store the resulting electrons. CCD was invented in 1969 at Bell Labs, ${ }^{6}$ and received good attention in the following years. Reducing camera noise is a key strategy to make CCD suitable for low-light detection. Cooling the CCD in a hermetic vacuum chamber dramatically reduces the dark current noise (a component of the camera noise), thus enhancing the sensitivity. The potential of CCD working in low-light conditions was realized about 10 years after the invention and yielded a noise equivalent signal of 16 electrons at $-50^{\circ} \mathrm{C}$. 7

To detect extremely weak signals, a vacuum tube device called image intensifier was optically attached to the front of a CCD and thus invented a new type of CCD called ICCD. ICCD magnifies the signal, reduces effectively the readout noise, thus improves naturally the detectability (i.e., the smallest photon signal that can be detected). ${ }^{1}$ ICCD cameras are superb in providing high temporal resolution (via the fast gating capability of the intensifiers) as well as single-photon detection and/ or photon counting capability. ${ }^{8}$ ICCD was commercially available in 1995.

EMCCD was invented in the 1990s, and commercially available in 2000. Nowadays, EMCCD is the most popular choice for low-light detection. EMCCD employs a special component called electron multiplication (EM) register, which is added between the readout register and the output amplifier in a frame-transfer CCCD, to amplify the signal. This signal multiplication effectively reduces read noise to undetectable levels, but introduces an additional noise factors (called excess noise) which halves the effective quantum efficiency (QE). ${ }^{9}$

(CMOS) appeared in the $1960 \mathrm{~s},{ }^{10}$ but had been too noisy and less sensitive than CCD for a long time. CMOS shares little design principle with CCD except for the use of photodiode in a pixel. Unlike CCD which stores and transfers the accumulated electrons in a pixel, CMOS immediately converts the electrons to a voltage by column amplifiers locating next to the photodiode. Owing to the heavy demand of cameras with lowcost, small size and low energy consumption in the consumer market (for example, cell phone, and digital camera), the imaging performance of CMOS has been improving rapidly. ${ }^{11}$ A new type of low-light detector, sCMOS, which is based on the CMOS technology, was announced in 2009. sCMOS offers low noise, high speed, and wide pixel arrays simultaneously, and thus offers great potentials for low-light detection. ${ }^{12}$ This new type of low-light camera has attracted great attention ever since its announcement. The capabilities of sCMOS in various applications, including super-resolution localization microscopy, ${ }^{13-15}$ have been tested and reported in the past several years.

\subsection{Current status of low-light cameras: examples and comparison}

It would be interesting to look into the specifications of several representative low-light cameras currently available in the market (Table 1). The Hamamatsu ORCA-R2 CCD, a widely used cooled CCD in laboratories, offers simultaneously high sensitivity (70\% QE), low read noise $\left(6 \mathrm{e}^{-}\right)$, and fast readout speed of $16.2 \mathrm{fps}$ at a resolution of $1344 \times 1024$ pixels. This camera can be cooled down to $-40^{\circ} \mathrm{C}$ to provide significantly low dark current of 0.0005 electrons $/ \mathrm{pixel} / \mathrm{s}$, so that dark current is still insignificant even after minutes of exposure. Moreover, the small pixel size of this CCD camera $(6.45 \mu \mathrm{m})$ makes it easy to work with a single high numerical aperture (NA) 60x objective to give a satisfactory Nyquist resolution. Note that EMCCD or ICCD cameras would require a secondary magnification lens to guarantee the Nyquist resolution, 
and thus normally results in a reduced efficiency in light detection.

With the use of Gen 3 image intensifier, the Andor iStar 312T ICCD is capable of capturing fast events $(<2 \mathrm{~ns})$ with a low read noise of $5.4 \mathrm{e}^{-}$and a good QE of $47.5 \%$, corresponding to a detectability of 57 photons. The Andor iXon Ultra 897 EMCCD offers the highest QE (95\%), the lowest read noise $\left(<1 \mathrm{e}^{-}\right)$and thus provides the best detectability among all of the cameras listed in Table 1. Clearly, this EMCCD would be the only choice when it is necessary to detect a signal with only a few photons. And, EMCCD is cheaper and has a longer life length when compared to ICCD.

Regarding the detectability, the sCMOS cameras listed in Table 1 seem to fit nicely into the gap between EMCCD and CCD/ICCD cameras. Moreover, these sCMOS cameras provide several extra advantageous features, including a small pixel size, large array of active pixels, and fast frame rate. Therefore, among all of the listed cameras, sCMOS is the best choice in applications which demand high imaging throughput at low-light levels. However, as we will discuss later, sCMOS has an inherent structure imperfection: each individual pixels in an sCMOS camera has its own characteristics (sensitivity, read noise, and offset), resulting in a troublesome image nonuniformity (pixel-to-pixel variation). A careful calibration on the imaging performance of individual pixels in an sCMOS camera would thus be beneficial to minimize the effects from such image nonuniformity.

It is worthwhile to note that the frame rates of a camera (see Table 1) estimates how fast an image can be continuously read out and saved in that camera, while another useful parameter called temporal resolution usually corresponds to the fastest exposure time of that camera. For most lowlight cameras, the temporal resolution is normally inversely proportional to the frame rate, assuming that the camera is working in a continuous imaging mode. However, for ICCD cameras, the fastest temporal resolution is defined by the shortest effective exposure time which is limited by the gating speed of the intensifier in that ICCD camera (for example, $2 \mathrm{~ns}$ for Andor iStar 312T). Note that ICCD cameras normally operate at a significantly longer exposure time (millisecond level) than the gate time (nanosecond level), although the signal is generated within such a fast gate time (thus called effective exposure time) and the camera is simply accumulating dark noise in the rest of the exposure time. ICCD camera is superb in imaging discrete fast events, but not in the frame rate.

\subsection{Key parameters for characterizing low-light cameras}

It is important to investigate the imaging performance of a camera before using it for any purpose. Some of the key parameters for characterizing lowlight cameras are discussed below.

- Pixel size: The dimensions of a pixel in a sensor, usually in $\mu \mathrm{m}$. The pixel size divided by the total magnification of a microscope system should be smaller than the required Nyquist resolution.

- Active pixels: The number of pixels in both $X$-and $Y$-directions which can be used to capture image. A bigger number of active pixels corresponds to bigger field-of-view.

Table 1. Specifications of several representative low-light cameras (as of February 2016).

\begin{tabular}{|c|c|c|c|c|c|c|c|}
\hline Model (Manufacturer) & Type & Active pixels & Pixel size (um) & $\begin{array}{l}\text { Read noise } \\
\left(\mathrm{e}^{-}, \mathrm{RMS}\right)^{\mathrm{a}}\end{array}$ & $\begin{array}{c}\text { Frame } \\
\text { rate }(f p s)^{b}\end{array}$ & $\mathrm{QE}_{\max }(\%)^{\mathrm{c}}$ & 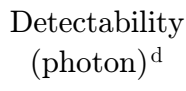 \\
\hline ORCA-R2 (Hamamatsu) & CCD & $1344 \times 1024$ & $6.4 \times 6.45$ & 6 & 16.2 & 70 & 43 \\
\hline iStar $312 \mathrm{~T}$ (Andor) & ICCD & $512 \times 512$ & $24 \times 24$ & 5.4 & 15.8 & 47.5 & 57 \\
\hline iXon Ultra 897 (Andor) & EMCCD & $512 \times 512$ & $16 \times 16$ & $<1^{\mathrm{e}}$ & 56 & 95 & $1.8^{\mathrm{f}}$ \\
\hline $\begin{array}{l}\text { ORCA-Flash4.0 V2 } \\
\text { (Hamamatsu) }\end{array}$ & sCMOS & $2048 \times 2048$ & $6.5 \times 6.5$ & 1.6 & 100 & 82 & 9.8 \\
\hline Zyla 5.5 (Andor) & sCMOS & $2560 \times 2160$ & $6.5 \times 6.5$ & 1.7 & 102 & 60 & 15 \\
\hline
\end{tabular}

${ }^{a}$ Read noise at fastest frame rate; ${ }^{b}$ Maximum frame rates (frame per second, fps) at full field of view. ${ }^{\mathrm{c}}$ Maximum $\mathrm{QE}$; ${ }^{\mathrm{d}} \mathrm{The}$ detectability was calculated by $\left(\mathrm{RN}^{*} 5\right) / \mathrm{QE}$, after considering that peak-to-peak noise is $\sim 5$ times the RMS value. ${ }^{16}$ Here RN is the read noise; ${ }^{\mathrm{e}} \mathrm{With}$ EM gain; ${ }^{\mathrm{f}}$ Assuming an effective read noise of $0.3 \mathrm{e}^{-}$for EM gain of 300 . Note that the effective read noise is almost linear to the EM gain value, and detecting single photon is possible if a high gain (for example, gain = 500) is used. The specifications were from the official websites of the camera manufacturers. 
- Frame rate: An estimation on how fast an image can be continuously read out and saved. The frame rate is determined principally by the number of pixels and the pixel read out rate; therefore, reading out only a sub-region of an image or pixel binning is helpful for improving the frame rate.

- Quantum efficiency: Equals to the percentage of incident photons that are converted to electrons by the sensor. QE varies as a function of the wavelength of the incident light. QE is extremely important in low-light detection as the signal from sample is very limited.

- Shot noise: May be also called as Poisson noise or photon noise. Shot noise arises from the phenomenon that the arrival of photons at the camera sensor is a stochastic process. Shot noise follows Poisson counting statistics and equals to square root of the input signal. Owing to its physical essence, shot noise can never be eliminated. ${ }^{17}$

- Read noise: Specifies the minimum operating noise in camera. Read noise increases with readout speed, but is independent on the input signal and/or exposure time. The read noise in CCD is the same for different pixels, while the read noise in CMOS is pixel-dependent.

- Dark noise: Camera noise that comes from thermally generated electrons; Dark noise increases with exposure time but is normally negligible when exposure time is short (minutes or less) for a cooled camera.

- Fixed pattern noise (FPN): Arises from the sensitivity difference of the pixels in a sensor. FPN has a spatially consistent influence from image to image and increases linearly with the signal. The FPN in CMOS cameras is typically more severe than that in CCD cameras.

- Excess noise: Originates from the EM process in EMCCDs. When a multiplication register is used to magnify a signal, it is impossible to have the same value in the total multiplication gain due to the uncertainty in the impact ionization process. Both dark and photon-generated signal in the camera are enlarged by the excess noise, thus a noise factor $\left(F_{n}\right)$ is introduced to describe the relationship. CCD and CMOS do not have excess noise because both of them do not have EM gain.

- Full well capacity (FWC): The maximum number of electrons a pixel can hold before saturation.
When it is near saturation, a camera starts to deviate from a linear response, thus diminishing the quantitative capability of the camera.

- Dynamic range (DR): The ratio of the theoretical maximum measurable signal to the minimum measurable signal. Since the FWC sets the upper limit and the read noise sets the lower limit, DR is usually expressed as the ratio of FWC and read noise.

- Camera conversion factor (Kadc): converts the digital number (in analog-to-digital unit, (ADU)) to electron, usually expressed in $\mathrm{e}^{-} / \mathrm{ADU}$.

\subsection{Signal-noise-ratio (SNR)}

SNR is widely used to determine the quality of an image or the imaging performance of a camera. SNR is defined as the signal divided by the total noise. If the SNR of an image is less than one, the signal of the image will be hard to recognize from the background. For low-light camera, there are mainly three types of noise sources: read noise, shot noise and FPN. ${ }^{18}$ Since these noises are independent to each other, the total noise is determined by

$$
\sigma_{\text {Total }}^{2}=\sigma_{\text {Shot }}^{2}+\sigma_{\text {Read }}^{2}+\sigma_{\mathrm{FP}}^{2}
$$

Here, $\sigma_{\text {Total }}, \sigma_{\text {Shot }}, \sigma_{\text {Read }}$, and $\sigma_{\mathrm{FP}}$ is the total noise, shot noise, read noise and fixed pattern noise, respectively. These noises have the same unit $\left(\mathrm{e}^{-}\right)$. Note that in real applications, the total noise may also contain shot noise introduced by photon background (such as stray light, or background fluorescence). The SNR is calculated as

$$
\mathrm{SNR}=\frac{S \times \mathrm{QE}}{\sigma_{\text {Total }}} .
$$

Here, $S$ is the number of collected signal (unit: photon). Note that for EMCCD working with a certain EM gain, the read noise usually equals to the read noise with no EM gain divided by the EM gain value used. However, as described before, the EM process introduces an additional noise called excess noise, which would double shot noise or halve QE.

For an ideal camera, the QE equals to 1, and there is only shot noise resulting from the input signal. Then the SNR of this ideal camera is:

$$
\mathrm{iSNR}=\sqrt{S}
$$


If we define a relative SNR (rSNR) as the SNR ratio between a real camera and an ideal camera:

$$
\mathrm{rSNR}=\frac{S \times \mathrm{QE}}{\sqrt{S \times \sigma_{\text {Total }}^{2}}} .
$$

Note that rSNR curve can make the difference among cameras more visible than the corresponding SNR curve.

\section{Understanding and using PTC for Camera Characterization}

\subsection{What is PTC?}

When choosing a camera for a specific application, reading the camera's technical specification sheet is just the first step. The data on the sheet represents only a best or average performance for this type of cameras. And, a camera can behave differently when the operating conditions are changed. Therefore, finding a quantitative approach to assess the performance of a specified camera in a certain environment is meaningful. Photon transfer curve (PTC) method is indispensable for this purpose.

PTC is a log-log plot of the total noise as a function of signal intensity (see Fig. 1). Note that the data used in Fig. 1 were simulated as follows: (1) We simulated three $128 \times 128$ images which mimic the read noise map, offset map and gain map of a real sCMOS camera (see description in Sec. 3), respectively. (2) We obtained a total of 50 different intensity levels ranging exponentially from 0.1 to 65,535 (in unit of DN). (3) For each intensity level, a Poisson noise generator was used to add shot noise

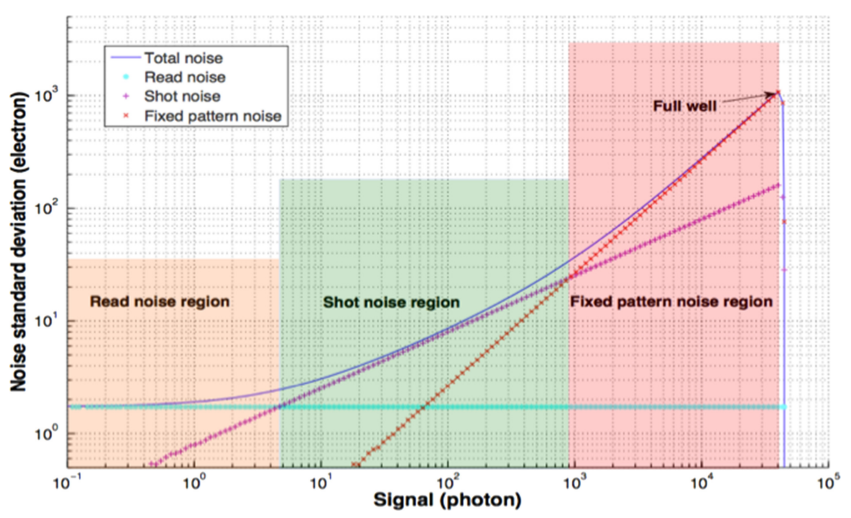

Fig. 1. A simulated PTC. Three noises can be seen as the dominant source at different signal levels: read noise, shot noise, and FPN. to each pixel, then the read noise was added using a Gaussian noise generator where the mean is zero for all pixels and the standard deviation of each pixel is from the read noise map. The resulted image was multiplied by the gain map to convert the pixel values from electron to $\mathrm{DN}$, and then was added with the offset map. We generated 100 image frames for each intensity level. (4) The simulated images after adding different noises were saved into a 16 bits integer TIFF image. After repeating the procedures for all the intensity levels, the simulated images were analyzed by PTC methods and the result were used to plot Fig. 1.

When the input signal increases, the dominant noise source changes from read noise to shot noise, and then FPN. Finally, the signal is saturated and a dramatic drop can be seen in the PTC curve.

The PTC method is mainly used to assess a camera by presenting many key parameters including shot noise, read noise, FPN, FWC and DR. An additional benefit from PTC method is that the Kadc can be determined precisely. ${ }^{17}$

\subsection{Experimental setup for PTC measurement}

A typical system for PTC measurement is shown in Fig. 2. A halogen lamp (or LED) is driven by a constant current source (fluctuation $<0.1 \%$ ), and the light emitted from the lamp is collected and collimated by a collector lens. A diaphragm is

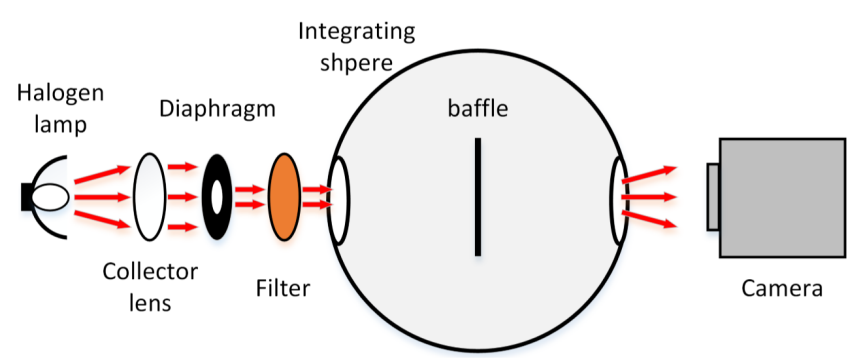

Fig. 2. A custom-build optical system for PTC measurement. The light from a halogen lamp (EJL 13164 24V200W Philips) is collected by a quartz lens with focal length of $30 \mathrm{~mm}$ (Spectral Analysis, China), filtered by either a $20 \mathrm{~nm}$ bandpass filter centered at $698 \mathrm{~nm}$ or a $40 \mathrm{~nm}$ bandpass filter centered at $594 \mathrm{~nm}$ (both from Mega-9, China), then entered a $30 \mathrm{~cm}$ diameter commercial integrating sphere (Flight technology, China). The camera under investigation is placed $32 \mathrm{~cm}$ away from the exit port ( $8 \mathrm{~cm}$ diameter) of the integration sphere, and a heavy duty lab jack (L490/M, Thorlabs) is used to align the camera. 


\section{Li et al.}

used to control the light intensity, while an optical filter is used to select light with specific wavelength, before entering into an integrating sphere. Note there is a baffle in the middle of the integrating sphere to prevent the light from passing directly through the sphere. The camera under studied is aligned to the exit port of the integrating sphere. The distance between the integrating sphere and the camera should be sufficient to obtain a uniform illumination ( $>99 \%$, see below). Special efforts must be taken to block the ambient light from reaching the low-light camera, since the camera has typically high light sensitivity.

Before PTC measurement, the spatial uniformity of the illumination must be checked carefully. The spatial uniformity is defined as the ratio between the lowest luminance and the mean luminance ${ }^{19}$ :

$$
U=\frac{E_{\text {min }}}{E_{\text {mean }}} .
$$

A more precise value in the uniformity can be obtained from an image that is averaged from more than 100 raw images where the image offset is already subtracted. For a good PTC measurement, the spatial uniformity should be above $99 \%$, otherwise the measurement of FPN would be imprecise. ${ }^{17}$

The illuminating uniformity is mainly influenced by the distance between the sensor and the exit port of the integrating sphere, and also the relative size of the sensor to the exit port diameter. ${ }^{20}$ Longer distance leads to larger illumination area with good uniformity, but dimmer illumination. On the other hand, it is better to use an integration sphere whose exit port is larger than the sensor. In practice, the uniformity is also influenced by the ambient light and the light reflected from the camera and the sphere body. Moreover, the temporal stability of the lamp should also be well maintained since the luminance fluctuation would introduce error in shot noise measurement. We recommend to use a lamp with intensity fluctuation smaller than $0.1 \%$.

\subsection{PTC measurement procedures}

Detailed procedures and explanations for PTC measurement have been well documented in the book written by Janesick and several other literatures. ${ }^{17,18}$ Basing on our previous works, ${ }^{13,21}$ here we present briefly such procedures to help readers understand the PTC method.

\subsubsection{Measuring image offset and read noise}

A total number of 100 successive raw image frames are captured without any incident light. Then, image offset (or called dark signal nonuniformity, DSNU) can be calculated as follows. For sCMOS, each pixel has an independent offset value:

$$
\mathrm{OFF}_{i}=\frac{\sum_{j=1}^{100} X(j)_{i}}{100}
$$

For EMCCD, all pixels have the same offset value:

$$
\mathrm{OFF}=\frac{\sum_{j=1}^{100} \sum_{i=1}^{N p} X(j)_{i}}{100 \times N p}
$$

where $X(j)_{i}$ is the raw grey value of the $i$ th pixel in the $j$ th frame, and $N p$ is the total number of pixels in a single image frame. Note that image offset is a bias that should be subtracted before estimating the real input signal and the noises.

Also, the spatial read noise is calculated from the difference of adjacent images, according to the following equation:

$$
\begin{aligned}
\sigma_{\text {Read, DN }}^{2}= & \sigma_{\text {Differenced Frame, DN }}^{2} \\
= & \frac{\sum_{i=1}^{N p} \sum_{j=1}^{99}\left[X(j)_{i}-X(j+1)_{i}\right]^{2}}{N p \times 2 \times 99}, \\
& \text { Spatial read noise }=\sigma_{\text {Read, DN }} .
\end{aligned}
$$

Note that the subtraction doubles the noise variance and removes the image offset. Additionally the subscript DN means the gray value. The temporal read noise of each pixel can be calculated as the standard deviation of the temporal fluctuation of the pixel value:

$$
\sigma(i)_{\text {Read, DN, temporal }}^{2}=\frac{\sum_{j=1}^{100}\left[X(j)_{i}-\mathrm{OFF}_{i}\right]^{2}}{99}
$$

The spatial read noise is a synthetical read noise value of all pixels, while the temporal read noise is the read noise of each pixel. Actually, the spatial read noise equals to the root mean square (RMS) value of the temporal read noises from all pixels (see below).

After rewriting Eq. (8), the spatial read noise can be calculated as:

$$
\begin{aligned}
\sigma_{\text {Read, DN }}^{2} & =\frac{\sum_{j=1}^{99} \sum_{i=1}^{N p}\left[X(j)_{i}-X(j+1)_{i}\right]^{2}}{2 \times 99 \times N p} \\
& =\frac{\sum_{i=1}^{N p}}{N p} \frac{\sum_{j=1}^{99}\left[X(j)_{i}-X(j+1)_{i}\right]^{2}}{2 \times 99}
\end{aligned}
$$


By the error propagation theory ${ }^{22}$ the variance of subtracted result (zero mean) divided by 2 equals to the variance without subtraction. Therefore,

$\frac{\sum_{j=1}^{99}\left[X(j)_{i}-X(j+1)_{i}\right]^{2}}{2 \times 99}=\frac{\sum_{j=1}^{100}\left[X(j)_{i}-\mathrm{OFF}_{i}\right]^{2}}{99}$.

Here $X(j)_{i}$ and $X(j+1)_{i}$ are independent and have identical distribution, and the subtraction doubles the variance. Substituting Eq. (12) into Eq. (11) and considering the definition in Eq. (10), we have:

$$
\sigma_{\text {Read, DN }}^{2}=\frac{\sum_{i=1}^{N p} \sigma(i)_{\text {Read, DN, temporal }}^{2}}{N p} .
$$

From Eq. (13), it is clear that the spatial read noise is the RMS value of the temporal read noise.

\subsubsection{Measuring total noise, shot noise, and FPN}

To measure the total noise, shot noise and FPN, the sensor should be exposed to the uniform light coming from the integrating sphere. Dozens of image groups under different light intensities are captured, and each group includes 100 image frames. Since the PTC is plotted in logarithmic coordination, the light intensity in these image groups should be adjusted exponentially (using a diaphragm) to make the final curves evenly sampled.

The total noise, shot noise, and FPN can be calculated sequentially from these images. First, the total noise is obtained by estimating the standard deviation of the pixels (after the offset is subtracted) in each image group:

$$
\begin{aligned}
\sigma_{\text {Total }, \mathrm{DN}}^{2} & =\sigma_{\text {Shot }, \mathrm{DN}}^{2}+\sigma_{\text {Read }, \mathrm{DN}}^{2}+\sigma_{\mathrm{FP}, \mathrm{DN}}^{2} \\
& =\frac{\sum_{j=1}^{100} \sum_{i=1}^{N p}\left[S(j)_{i}-M_{i}\right]^{2}}{99 \times N p},
\end{aligned}
$$

where $S(j)_{i}$ is the gray value of the $i$ th pixel in the $j$ th frame after the offset is subtracted. $M_{i}$ is the mean value of $S(j)_{i}$ averaged from the 100 image frames.

As the FPN is fixed within an image group, it can be eliminated by subtracting two successive images:

$$
\begin{aligned}
\sigma_{\text {Differenced Frame, DN }}^{2} & =\sigma_{\text {Shot, DN }}^{2}+\sigma_{\text {Read, DN }}^{2} \\
& =\frac{\sum_{j=1}^{99} \sum_{i=1}^{N p}\left[S(j)_{i}-S(j+1)_{i}\right]^{2}}{2 \times 99 \times N p}
\end{aligned}
$$

Then,

$$
\begin{gathered}
\sigma_{\text {Shot, DN }}^{2}=\sigma_{\text {Differenced Frame, DN }}^{2}-\sigma_{\text {Read, DN }}^{2} . \\
\sigma_{\mathrm{FP}, \mathrm{DN}}^{2}=\sigma_{\text {Total, DN }}^{2}-\sigma_{\text {Differenced Frame, DN }}^{2} .
\end{gathered}
$$

Furthermore, since the FPN originates not only the pixel size mismatch but also the parallel read out architecture in the column, the FPN can be separated into pixel FPN (pFPN) and column FPN $(\mathrm{cFPN})$. The $\mathrm{pFPN}$ characterizes the pixel size mismatch, while the $c F P N$ is related to the stripes in the captured images. Two steps are used to determine the cFPN: (1) an averaged image from 100 raw image frames is acquired, then after the image offset is removed, the pixel values from the same column are averaged to present the mean value of the column; then (2) the standard deviation of the mean values from all columns is calculated to give the $c F P N$ value. Note that read noise, shot noise and pixel FPN are all minimized by averaging.

After the cFPN is obtained, the pFPN is calculated based on:

$$
\sigma_{\mathrm{FP}, \mathrm{DN}}^{2}=\sigma_{p \mathrm{FP}, \mathrm{DN}}^{2}-\sigma_{c \mathrm{FP}, \mathrm{DN}}^{2} .
$$

The input light intensity in unit DN can be calculated in each group as

$$
\operatorname{Signal}_{\mathrm{DN}}=\frac{\sum_{j=1}^{100} \sum_{i=1}^{N p} S(j)_{i}}{100 \times N p} .
$$

\subsubsection{Measuring camera conversion factor}

Kadc is required for converting the unit of the signal from digital number to electron. Because shot noise in unit $\mathrm{e}^{-}$is equal to the square root of signal intensity in unit $\mathrm{e}^{-}$, a formula for determining Kadc can be derived as:

$$
\operatorname{Kadc}\left(e^{-} / \mathrm{DN}\right)=\frac{\operatorname{Signal}_{\mathrm{DN}}}{\sigma_{\text {Shot, DN }}^{2}} .
$$

The precision in Kadc measurement can be improved by least-squares fitting to the Kadc values from different illumination intensities. And, the obtained Kadc value is used to convert the unit of the noises:

$$
\begin{aligned}
\text { Total noise } & =\operatorname{Kadc}\left(e^{-} / \mathrm{DN}\right) \times \sigma_{\text {Total, DN }}, \\
\text { Read noise } & =\operatorname{Kadc}\left(e^{-} / \mathrm{DN}\right) \times \sigma_{\text {Read, DN }}, \\
\text { Shot noise } & =\operatorname{Kadc}\left(e^{-} / \mathrm{DN}\right) \times \sigma_{\text {Shot, DN }},
\end{aligned}
$$

Fixed Pattern noise $=\operatorname{Kadc}\left(e^{-} / \mathrm{DN}\right) \times \sigma_{\mathrm{FP}, \mathrm{DN}}$. 


\section{Characterizing Low-Light Cameras Beyond PTC}

Although the PTC method is an excellent tool to access different low-light cameras, it is still a synthetical method which provides only the performance of an entire camera. As we discussed earlier, sCMOS camera is a promising camera for low-light detection. However, unlike CCD cameras, each individual pixels in an sCMOS camera have their own characteristics. This indicates that accessing only the performance of an entire sCMOS camera is not sufficient for quantitative imaging applications. Therefore, it is necessary to perform additional characterizations to the sCMOS camera at single pixel level, mainly including offset map, read noise map and gain map. In this section, we will discuss how to measure these maps and how precisely we can obtain for these maps.

Measuring these three maps (offset, read noise, and gain) can be performed with the optical system identical to the PTC measurement (Fig. 2), but with a much bigger number of raw images. In PTC measurement, all data from spatial and temporal pixels are used to calculate a single synthetical value. Therefore, a total of 100 raw images would be sufficient to achieve very high precision. However, to measure these maps, only temporal pixel values can be used to present pixel independent information. To ensure a sufficient measurement precision, thousands of raw images are commonly used.

\subsection{Measuring offset map and read noise map}

The offset map and read noise map are measured from image frames without incident light. First, close all room lights and capture at least 1000 frames of raw images. Then the read noise map is the standard deviation of each pixel along time ${ }^{14}$ :

$$
\sigma_{i \text {,read }}^{2}=\operatorname{var}_{i, \text { read }}=\frac{1}{N-1} \sum_{\mathrm{n}=1}^{N}\left(X_{i}^{n}-o_{i}\right)^{2}
$$

And offset map is the fixed image bias after the read noise is removed by average ${ }^{14}$ :

$$
o_{i}=\frac{1}{N} \sum_{n=1}^{N} X_{i}^{n} .
$$

Here, $X_{i}^{n}$ is the pixel value of pixel $i$ in the $n$th frame, $o_{i}$ is the offset value of pixel $i, \sigma_{i, \text { read }}$ and $\operatorname{var}_{i, \text { read }}$ are the read noise (standard deviation) and read noise variance of pixel $i$ respectively.

\subsection{Measuring gain map}

The gain is sensitive on the process of converting electron to gray value. The gain map measurement is theoretically based on the intrinsic characteristic of photon (i.e., the shot noise in unit $\mathrm{e}^{-}$is equal to the square root of input signal in unit $\mathrm{e}^{-}$). This provides a method to gauge the gain by:

$$
\text { gain }=\frac{\operatorname{var}_{\text {shot }}}{\text { Signal }} \text {. }
$$

Here $\operatorname{var}_{\text {shot }}$ is the variance of shot noise, Signal is the input light intensity, both in unit DN. To ensure a high measurement precision, several groups of raw images are captured and the final gain for a pixel is obtained by a least-squares fitting:

$$
\operatorname{gain}_{i}=\frac{\sum_{m=1}^{M} \operatorname{var}_{i, \text { shot }}^{m} \times \operatorname{Signal}_{i}^{m}}{\sum_{\mathrm{m}=1}^{M}\left(\text { Signal }_{i}^{m}\right)^{2}}
$$

And

$$
\begin{gathered}
\operatorname{var}_{i, \text { shot }}^{m}=\operatorname{var}_{i, \text { total }}^{m}-\operatorname{var}_{i, \text { read }}, \\
\operatorname{Signal}_{i}^{m}=\bar{S}_{i}^{m}-o_{i} .
\end{gathered}
$$

Here, $M$ is the total number of image groups for measuring the gain, and $\mathrm{m}$ indicates the $m$ th image group. The $\operatorname{var}_{i, \text { total }}^{m}$ is the total noise variance of pixel $i$ in the $m$ th image group, calculated as the variance of pixel value in images with input signal. $\operatorname{var}_{i \text { shot }}^{m}$ is the shot noise variance of pixel $i$ in the $m$ th image group. $\bar{S}_{i}^{m}$ is the mean value of pixel $i$ in the $m$ th image group after removing the offsets.

To measure the gain experimentally, the sensor should be exposed to a stabilized light $(<0.1 \%$ fluctuation) with different intensities, and hundreds of thousands of raw image frames are captured under each light intensity. Additionally, for a convenient discussion on the measurement precision later, we assume the light intensity for group $m$ is $m^{*} D$. Here, $D$ is the light intensity increasing between adjacent groups, and $M^{*} D$ achieves several thousands of photons.

\subsection{Precision analysis on the measured maps}

The precision we discuss here is the standard deviation of the measured value, and can be estimated 
through the error propagation theory. ${ }^{22}$ The precision of offset map can be deduced straightforward. Since the fluctuation of the image without light is caused by read noise, the precision of the offset measured from single image equals to the read noise in unit DN (referred to as $\sigma_{\text {read }}$ ). After averaged by $N$ image frames, the precision of the final offset map is ${ }^{22}$ :

$$
\sigma_{\text {offset }}=\frac{\sigma_{\text {read }}}{\sqrt{N}}
$$

It is hard to derive the precision of read noise and read noise variance (that is, the square of the read noise) directly from the error propagation theory. Instead, they can be derived from the probability distribution of standard deviation and variance, respectively, ${ }^{23,24}$ and the precision of the read noise and read noise variance are given $\mathrm{as}^{25}$ :

$$
\begin{gathered}
\sigma_{\text {std }} \approx \frac{\sigma_{\text {read }}}{\sqrt{2(N-1)}}, \\
\sigma_{\text {var }}=\frac{\sqrt{2} \sigma_{\text {read }}^{2}}{\sqrt{N-1}} .
\end{gathered}
$$

The precision for the gain map measurement can be deduced from the precision of the data used to obtain the gain. For a specific pixel, the precision of the input signal and the total noise variance are similar to the offset and read noise variance, after replacing the $\sigma_{\text {read }}$ with the standard deviation of total noise (in unit DN) in each image group. Then they are given as

$$
\begin{aligned}
& \sigma_{\mathrm{var}_{m, \text { total }}}= \frac{\sqrt{2} \mathrm{var}_{m, \text { total }}}{\sqrt{N-1}} \approx \frac{\sqrt{2} \operatorname{var}_{m, \text { shot }}}{\sqrt{N}} \\
&= \frac{\sqrt{2} \bar{g} \cdot \text { Signal }_{m}}{\sqrt{N}}, \\
& \sigma_{\text {Signal }}=\frac{\sigma_{\text {total }}}{\sqrt{N}} .
\end{aligned}
$$

For Eq. (29), the $\operatorname{var}_{\text {read }}$ and its precision are small compared with $\operatorname{var}_{m, \text { total }}$ and its precision, and thus can be neglected. Then the precision of $\operatorname{var}_{m, \text { shot }}$ is approximated by $\operatorname{var}_{m, \text { total }}$.

$$
\sigma_{\operatorname{var}_{m, \text { hhot }}} \approx \frac{\sqrt{2} \operatorname{var}_{m, \text { shot }}}{\sqrt{N}}=\frac{\sqrt{2} \bar{g} \cdot \text { Signal }_{m}}{\sqrt{N}} .
$$

We assume the light intensity for group $m$ is $m^{*} D$ (as described previously), that is

$$
\operatorname{Signal}_{m}=m * D \text {. }
$$

Then consider Eq. (28) and introduce the error propagation theory, the precision of gain can be given as

$$
\begin{aligned}
\sigma_{\text {gain }} & \approx \sqrt{\sum_{m=1}^{M} \frac{\operatorname{Signal}_{m}^{2}}{\left(\sum_{m=1}^{M} \text { Signal }_{m}^{2}\right)^{2}} \cdot\left(\frac{\sqrt{2} \bar{g} \cdot \text { Signal }_{m}}{\sqrt{N}}\right)^{2}} \\
& =\frac{\sqrt{2} \bar{g}}{\sqrt{N}} \cdot \sqrt{\frac{\sum_{m=1}^{M} m^{4}}{\left(\sum_{m=1}^{M} m^{2}\right)^{2}}}
\end{aligned}
$$

When $M$ is in the region from 10 to 40 (commonly used range), the precision of the gain is approximated as

$$
\sigma_{\text {gain }}=\frac{1.87 \bar{g}}{\sqrt{N M}} .
$$

Here $\bar{g}$ is the least-squares fitted gain value of each pixel, $N$ is the number of image frame in each group, and $M$ is total number of image groups used to fit the gain.

Precision analysis is useful to calculate the number of raw images necessary for a given measurement precision. Since each pixel has its own individual read noise and gain, different measurement precision can be derived for these pixels. Then an average or RMS value can be obtained to express the comprehensive precision, or just substitute the mean or RMS read noise, variance and gain into the precision formula. Another simple method to estimate the precision in a real experiment is repeating measurement of the map: (1) subtract two relevant maps; (2) calculate the standard deviation of the subtracted maps, and (3) divide the obtained standard deviation value by the square root of 2 . The value obtained from the last step estimates the RMS measurement precision of the corresponding map, as shown by the error propagation theory.

\section{Characterizing and Comparing the Performance of Low-Light Cameras}

\subsection{The same camera under different operation conditions}

As described above, PTC is a method for characterizing the camera noises under a certain operation condition, that is, a combination of many operation parameters (including but not limited to wavelength, pixel readout rate, digitization, etc.). When 


\section{Li et al.}

the operation condition changes, the imaging performance of the camera may also change, leading to a different PTC.

\subsubsection{The influence of wavelength}

It is wellknown that the sensitivity of a camera is wavelength-dependent, and QE is used to characterize such dependence: higher QE means higher sensitivity or signal. It is also wellknown that shot noise is linearly dependent on the square root of the signal, and FPN is linearly dependent on the signal itself. $^{18}$ For a Hamamatsu Flash 4.0 sCMOS camera under studied (Model: C11440-22CU, SN: 750939) according to the specifications from the manufacturer, the QE is 0.72 and 0.64 for $593 \mathrm{~nm}$ and $698 \mathrm{~nm}$, respectively.

The PTC results for these two wavelengths are shown in Figs. 3(a) and 3(b). Since the difference between Figs. 3(a) and 3(b) is hardly distinguishable, we calculated the corresponding rSNR curves (shown in Fig. 3(c)). Clearly from these rSNR curves, the sCMOS camera shows better imaging performance (higher rSNR) at the wavelength with higher QE (that is, $593 \mathrm{~nm}$ ). And, when the signal is between 40 and 1000 photons, the camera seems to be close to a hypothetical QE-limited camera which has the same QE as a real camera and has only shot noise. The rSNR gaps between a real camera and a

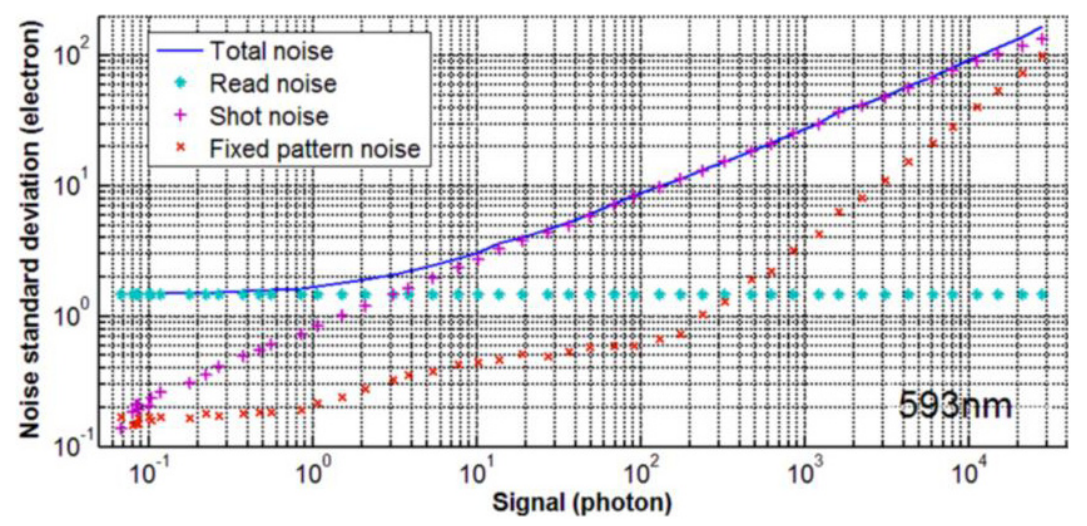

(a)

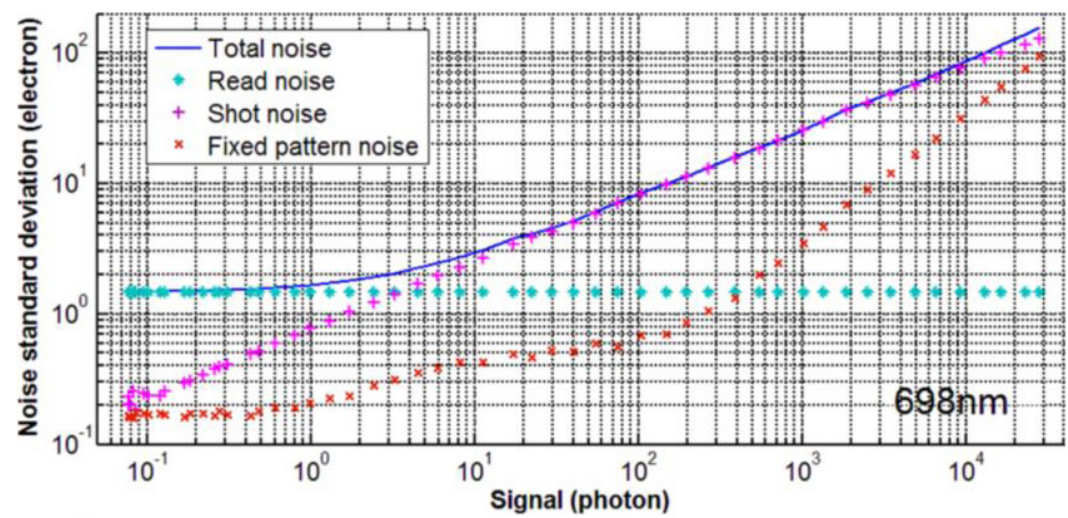

(b)

Fig. 3. The imaging performance of the same Hamamatsu Flash 4.0 sCMOS camera under two different wavelengths. The PTC results for $593 \mathrm{~nm}$ (a) and $698 \mathrm{~nm}$ (b), and the corresponding rSNR curves (c). The reference lines in (c) indicate the rSNR curves of two hypothetical QE-limited cameras (QE $=72 \%$ or $64 \%$ ) which have only shot noise. The red arrow in (c) shows a gap in the rSNR curves which is caused by the use of two different analog-to-digital converter. (d) The relative contributions of different noises to the total noise. Here the percentage is defined as the square of individual noise divided by the square of total noise. Note that the signal level below one photon means that there are only one photon occurred in several images, and that the rSNR of a hypothetical QElimited camera equals to the square root of the QE of that camera. 


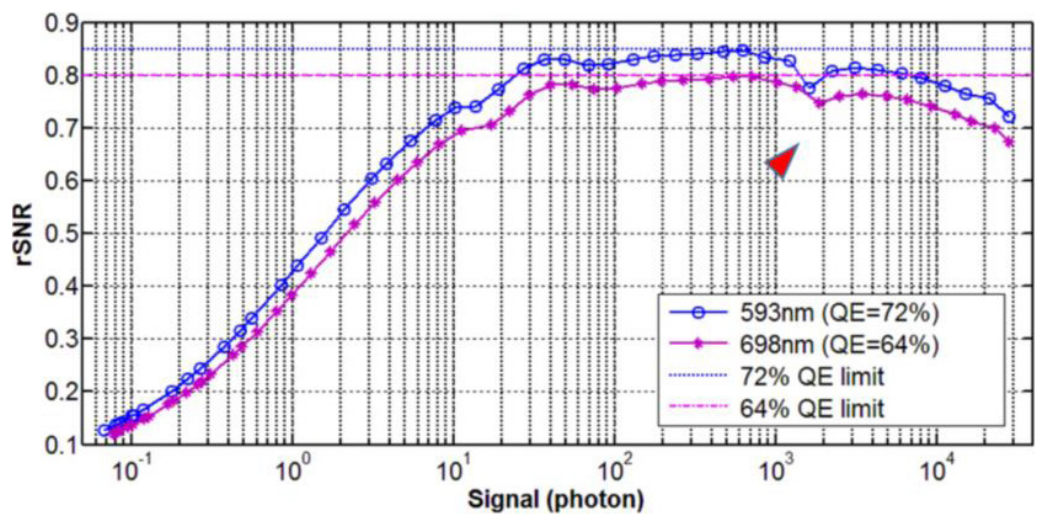

(c)

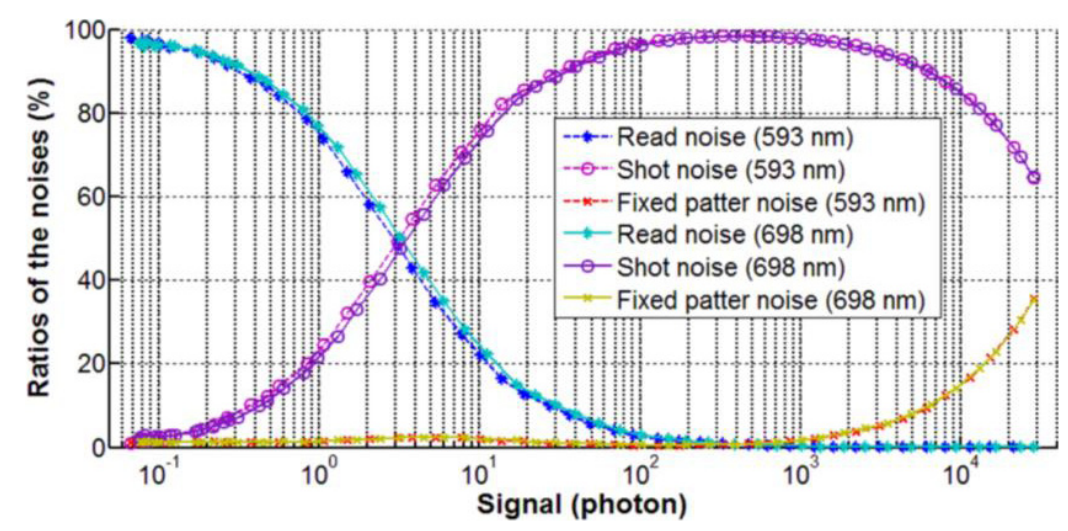

(d)

Fig. 3. (Continued)

hypothetical QE-limited camera highlight the impacts of read noise and FPN.

It is interesting to have a further look at the relative contribution of different noises to the total noise under different signal intensities (see Fig. 3 (d)). In the signal range between 30 and 7000 photons, the percentage of shot noise to total noise is $>90 \%$, indicating that the shot noise is the dominant noise source. The contribution from the read noise to the total noise decreases gradually as the signal gets stronger, and becomes negligible $(<10 \%)$ if the signal is $>40$ photons. Conversely, the contribution from the FPN gradually increases when the signal strengthens, and becomes important $(>10 \%)$ when the signal intensity is $>7000$ photons.

\subsubsection{The influence of EM gain}

Read noise is usually considered as the major factor which sets the detection limit of low-light cameras.
And, for the cameras working at fast read out rates $(5 \mathrm{MHz}$ or higher), the read noise is significantly higher, ${ }^{26}$ indicating that it is hard to make low-light cameras working at high imaging speed. As mentioned in Sec. 1.1, EMCCD cameras take advantage of EM to effectively eliminate read noise at high read out rates, therefore push the detection limit to single photon (see the note in Table 1). Here, we investigate how EM gains affects the imaging performance of a commercial Andor iXon 897 EMCCD camera (Model: DU-897D-C00-BV, SN: X-4652).

It is clear that higher EM gains compress more efficiently the read noises: $1.03 \mathrm{e}^{-}$for Gain $=50$, $0.56 \mathrm{e}^{-}$for Gain $=100$, and $0.34 \mathrm{e}^{-}$for Gain $=200$, respectively (Figs. 4(a)-4(c)). This finding is consistent to the literature. Interestingly, the rSNR curves (Fig. 4(d)) show that higher EM gain is preferable if the signal is ultra-weak (in our case, it is better to use Gain $=200$ when the signal is $<6$ photons), while a relative low EM gain is optimal when the signal is stronger (Gain $=50$ when the 


\section{Li et al.}

signal is $>23$ photons). Additionally, it is worthwhile to mention that the rSNR values of this EMCCD camera are always significantly smaller than that from a hypothetical QE-limited camera $(\mathrm{QE}=92 \%)$ which has only shot noise, showing a negative effect from the EM process.

\subsection{The temporal and spatial read noises of an sCMOS camera}

Temporal read noise, usually abbreviated as read noise, is the temporal intensity variation of a given pixel without input signal (Fig. 5(a)). It restricts the minimal signal a low-light camera can detect. ${ }^{27}$ As we described before, the temporal read noise of individual pixels is consistent for a CCD camera, but varies for an sCMOS camera. Practically, the read noise of a CCD camera can be characterized straightforwardly by the mean temporal read noise of all pixels. While for an sCMOS camera, the map of the temporal read noise of all pixels should be measured and the RMS value of the temporal read noise map (Fig. 5(b)) is calculated to present the overall read noise, as indicated in the datasheet of that camera.

A spatial read noise value can be calculated from the spatial intensity variation of all pixels, which takes the anisotropic temporal read noise into account. The spatial read noise is theoretically identical to the RMS value of temporal read noise map (see Sec. 2.3.1), and can be a good evaluation of the overall read noise of sCMOS camera. Note that the median value of the temporal read noises is sometimes reported as the read noise for some

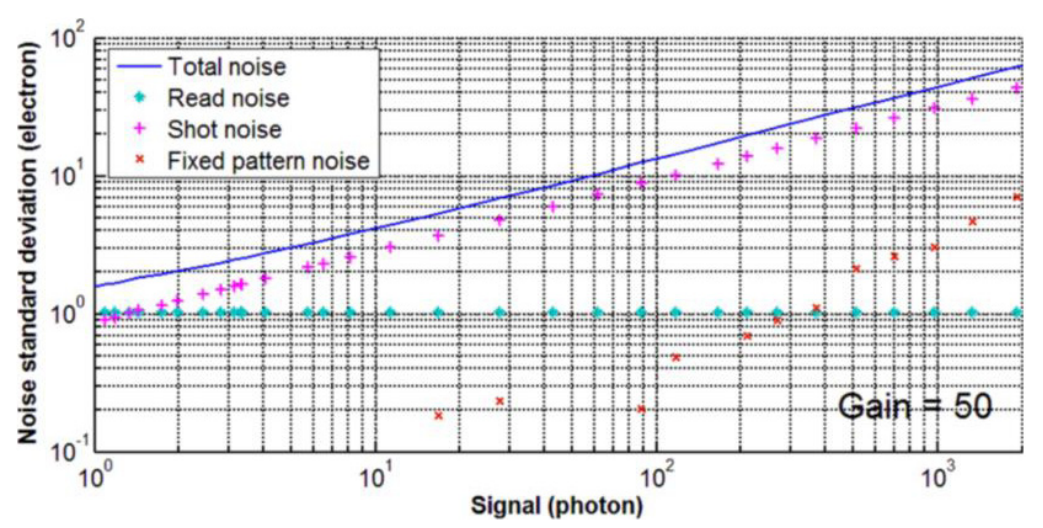

(a)

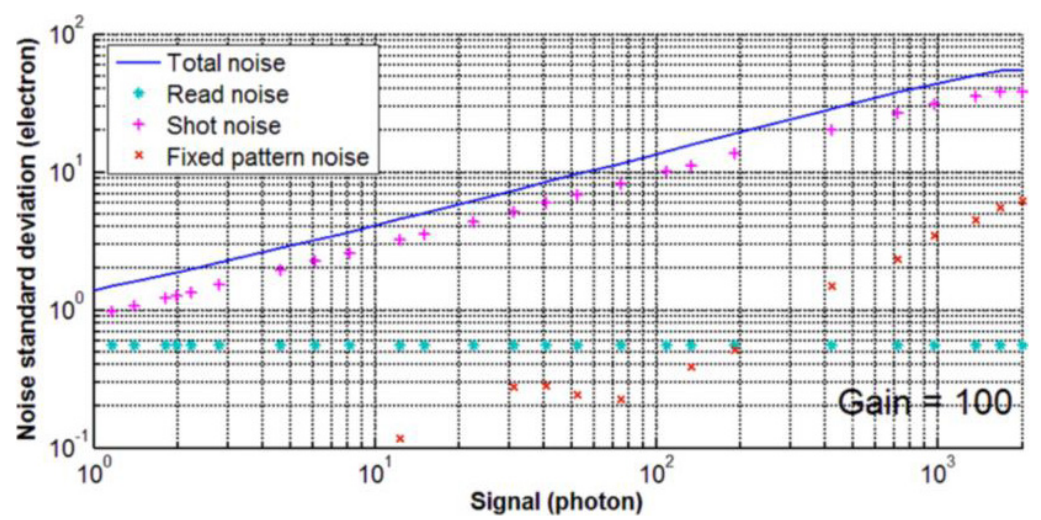

(b)

Fig. 4. EM gain influence on the imaging performance of the same Andor iXon 897 EMCCD camera. (a) Gain $=50$, (b) Gain $=100$ (b), (c) Gain = 200. (d) Theoretical and experimental rSNR curves for different EM gains. The inserted figure in (d) is an enlarged view of the region marked by the red rectangle in $(\mathrm{d})$. The measurement is performed with a narrow band light centered $698 \mathrm{~nm}$. Note that the read noise values from (a) to (c) decrease from $1.03 \mathrm{e}^{-}$(a) to $0.56 \mathrm{e}^{-}$(b), and then further to $0.34 \mathrm{e}^{-}$(c), and that the rSNR of a hypothetical QE-limited camera equals to the square root of the QE of that camera. 


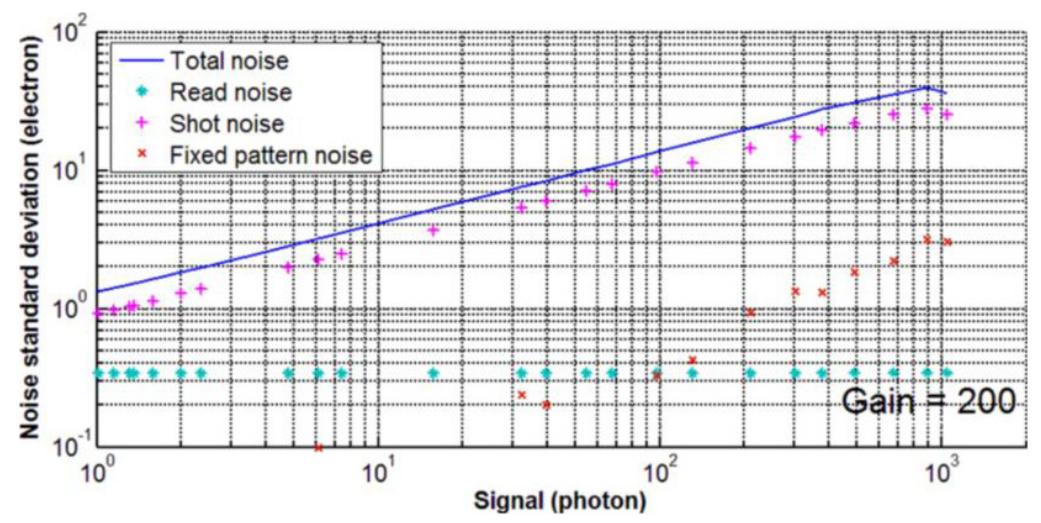

(c)

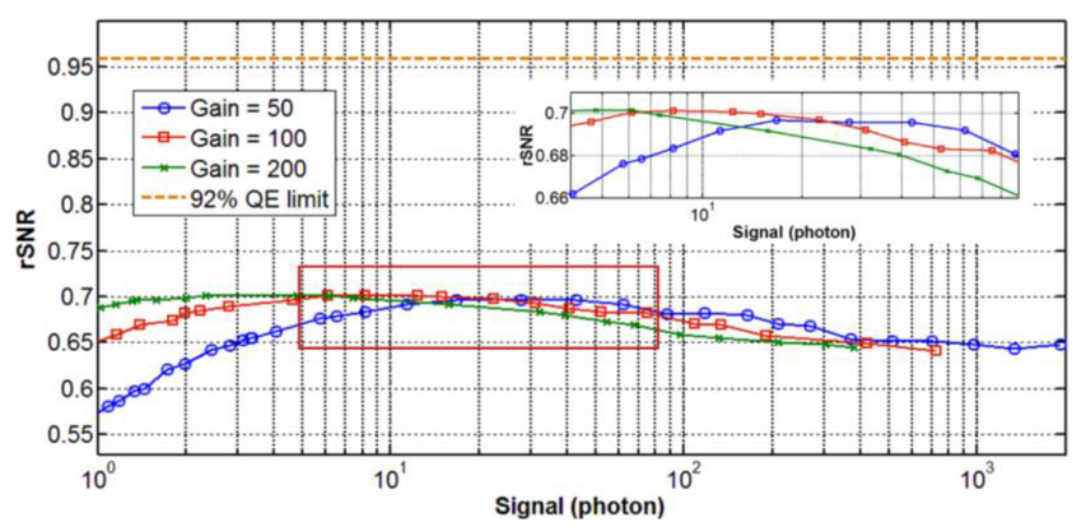

(d)

Fig. 4. (Continued)

commercial sCMOS cameras, ${ }^{28,29}$ and that the spatial read noise of a CCD or EMCCD camera has the same value as its temporal read noise.

Commercial sCMOS camera are usually equipped with a function called pixel correction or similar, which is used to correct those pixels with high temporal read noise. The distribution of the temporal read noise values of the Hamamatsu Flash 4.0 sCMOS camera is shown in Fig. 5(b). The read noise with pixel correction on is calculated to be $1.45 \mathrm{e}^{-} \mathrm{rms}$ for this camera, meaning that with this camera we should expect to see about 1.45 electrons of temporal read noise per pixel. Otherwise, the read noise with pixel correction off is $1.74 \mathrm{e}^{-}$. A long tail is seen in the distribution of the temporal read noise, especially with pixel correction off. Totally, $3.3 \%$ pixels with pixel correction on and $5.9 \%$ pixels with pixel correction off are found to exhibit temporal read noise of $3.0 \mathrm{e}^{-}$RMS or higher. The spatial read noise of this camera is $1.45 \mathrm{e}^{-}$, which is usually mentioned in the datasheets from the camera manufacturer with pixel correction on.

\subsection{Characterizing the pixel-dependent noises of a commercial $s C M O S$ camera}

Figure 6 presents the pixel-dependent noises (imaging uniformity) of the Hamamatsu Flash 4.0 sCMOS camera. The offset in this camera has a mean of $100.2 \mathrm{DN}\left(45.8 \mathrm{e}^{-}\right)$and a standard deviation of $0.54 \mathrm{DN}\left(0.25 \mathrm{e}^{-}\right)$(Fig. 6(b)) with $1.4 \%$ of pixels lying outside three standard deviations of the mean. The read noise is $3.82 \mathrm{DN}\left(1.74 \mathrm{e}^{-}\right) \mathrm{RMS}$, and $1.5 \%$ of pixels' read noise is higher than three times of the RMS value. Interestingly, stripes can be seen in the Gain map (Fig. 6(a)), showing the parallel column read out architectures of the sCMOS 


\section{Li et al.}

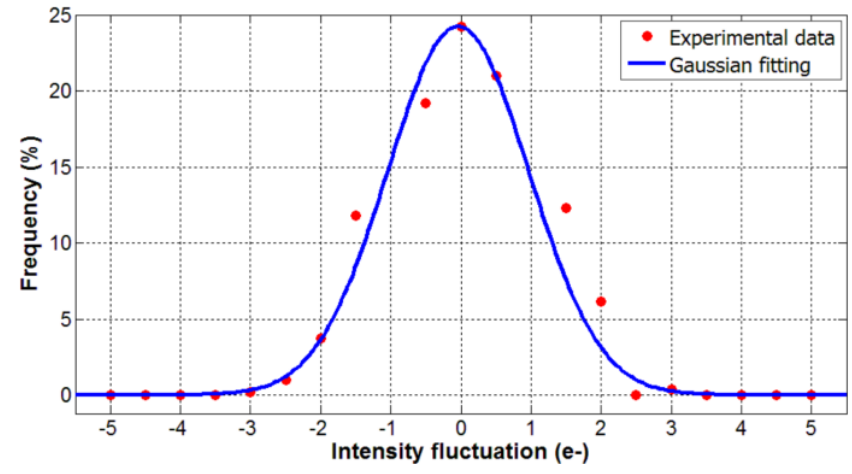

(a)

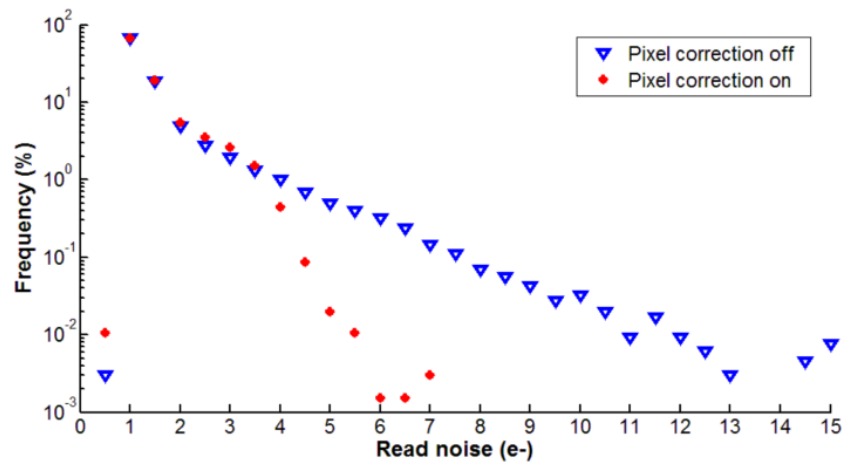

(b)

Fig. 5. The temporal read noises from the commercial Hamamatsu Flash 4.0 sCMOS camera. (a) The temporal intensity fluctuation histogram of a pixel after offset reduction. Note that the temporal read noise of the pixel is calculated by the standard deviation of the intensity fluctuation. Here the pixel correction is off. (b) The histogram of the temporal read noises from a $256 \mathrm{x} 256$ pixel region, with and without pixel correction.

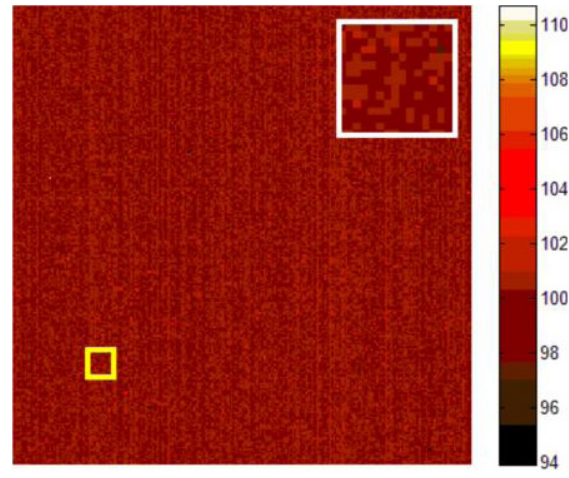

(a)

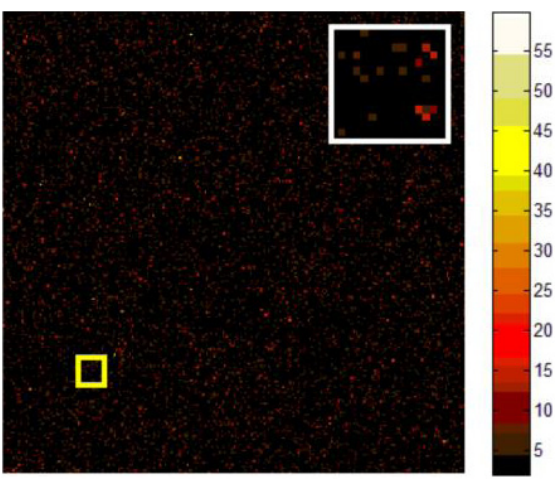

(c)

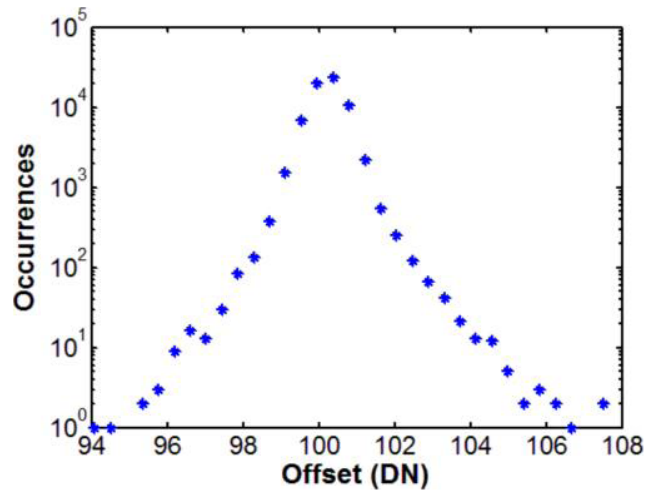

(b)

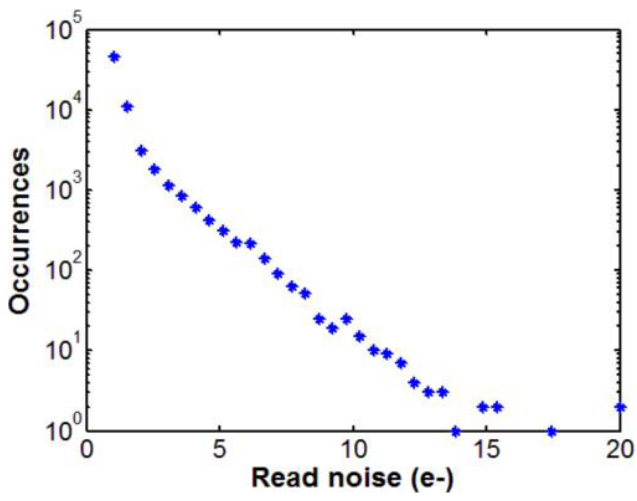

(d)

Fig. 6. The pixel-dependent noises of the Hamamatsu Flash 4.0 sCMOS camera. (a) The offset map and (b) the corresponding histogram. (c) The read noise map and (d) the corresponding histogram. (e) The Gain map and (f) the corresponding histogram. The enlarged images (15x15 pixels) in the upper right corners of (a), (c) and (e) show local fluctuations of the maps in the regions in the lower left. To measure the Gain map, the signal was linearly increased from 0 to 1000 photon/pixel, and a total of 16 image groups were captured with 1000 raw image frames in each group. The first group was measured with no incident light. A sub-region of $256 \times 256$ pixels was used for all measurements. 


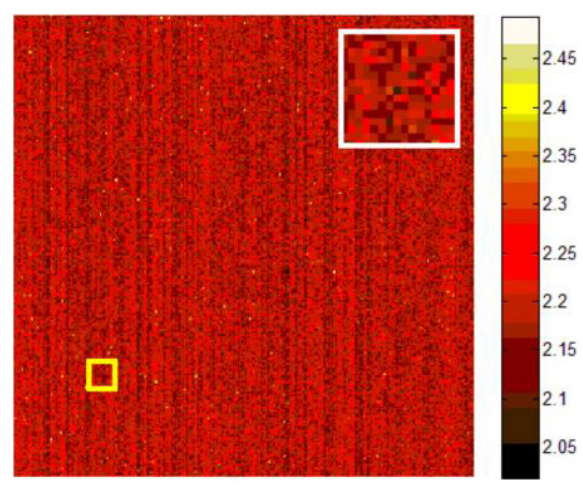

(e)

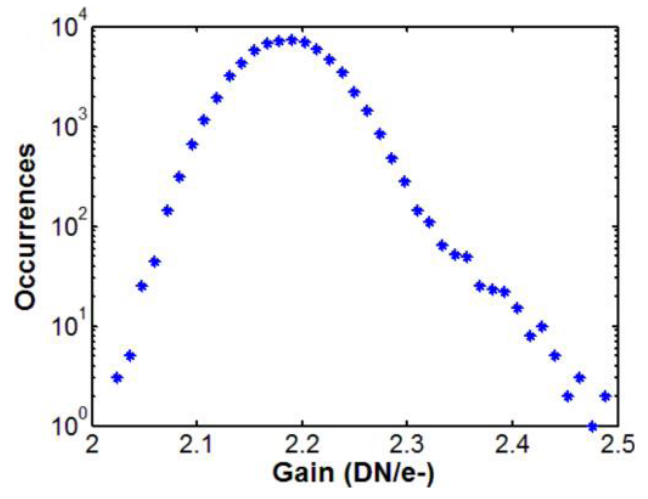

(f)

Fig. 6. (Continued)

camera. The gain has a mean of $2.19(\mathrm{DN} / \mathrm{e})$ and a standard deviation of 0.043 , and $0.6 \%$ of pixels lie outside three standard deviations of the mean. Note that the maps are measured without pixel correction since the correction can change the read noise distribution and may affect the quantitative analysis.

Additionally, it is beneficial to estimate the precision for the pixel-dependent noise measurement. Here, we calculate the synthetical precisions for each type of pixel-dependent noise using the method introduced in the last paragraph in Sec. 3.3, i.e., derived from the differenced maps. The precision values from both simulated and experimental data are shown in Table 2, along with the theoretical precision values calculated using Eqs. (28)-(31).

For the offset map and gain map, the simulated and experimental precision are very close to the corresponding theoretical values and agree well with

Table 2. Precision in the pixel-dependent noise maps.

\begin{tabular}{lccc}
\hline Type & Read noise & Offset & Gain \\
\hline Theoretical precision & 0.0854 & 0.1207 & 0.0334 \\
Simulated precision & 0.0856 & 0.1214 & 0.0338 \\
Experimental precision & 0.1282 & 0.1333 & 0.0339 \\
\hline
\end{tabular}

Notes: The units of these precision values are consistent with respective maps. To check the error propagation theory, we simulated two sets of images. In the simulation, a set of measured maps was used and the data were generated by the procedures described in Sec. 2.1, except that here 15 linearly distributed intensity levels with 1000 image frames in each intensity level were used. the precision theory. The simulated precision for the read noise is consistent with precision theory, but the precision of experimental value is a little higher than predicted, the reason is still under investigation.

\subsection{Comparing different low-light cameras}

It is often required to select a camera with best sensitivity from the market. The answer can be readily obtained from comparing the SNR or rSNR curves (Fig. 7). SNR curves are used more often in research papers or technical papers, but may not be efficient when the difference between two cameras is not very big (see Fig. 7(a)). Therefore, we recommend to use rSNR curves to provide a much better comparison for the small difference.

As seen from Fig. 7(b), when the signal intensity is $<7$ photon/pixel, the Andor iXon 897 EMCCD camera working with EM Gain $=200$ is the best choice (highest rSNR). If the signal is higher than 7 photon/pixel, we should definitely select the Hamamatsu Flash 4.0 sCMOS camera. Another interesting phenomena we can observe from Fig. $7(\mathrm{~b})$ is that when the signal intensity is $>16$ photon/pixel, the EMCCD working at low EM gain (Gain $=50)$ provides better sensitivity than the same camera working at a high EM gain (Gain = 200). Additionally, when the signal intensity is $>480$ photon/pixel, the EMCCD with EM Gain = 200 approaches nearly its saturation, although the rSNR seems to rise sharply. 


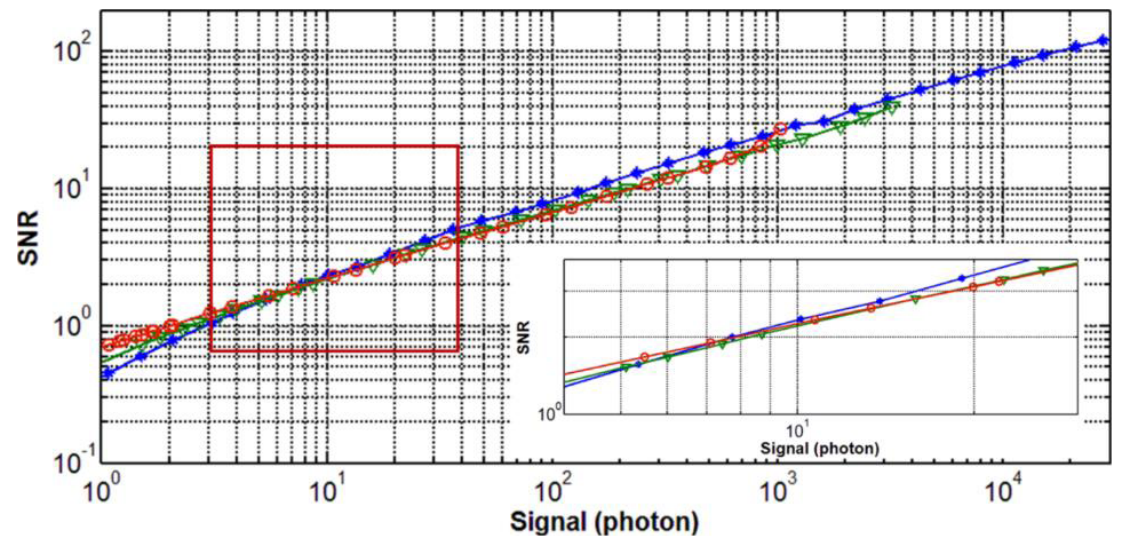

(a)

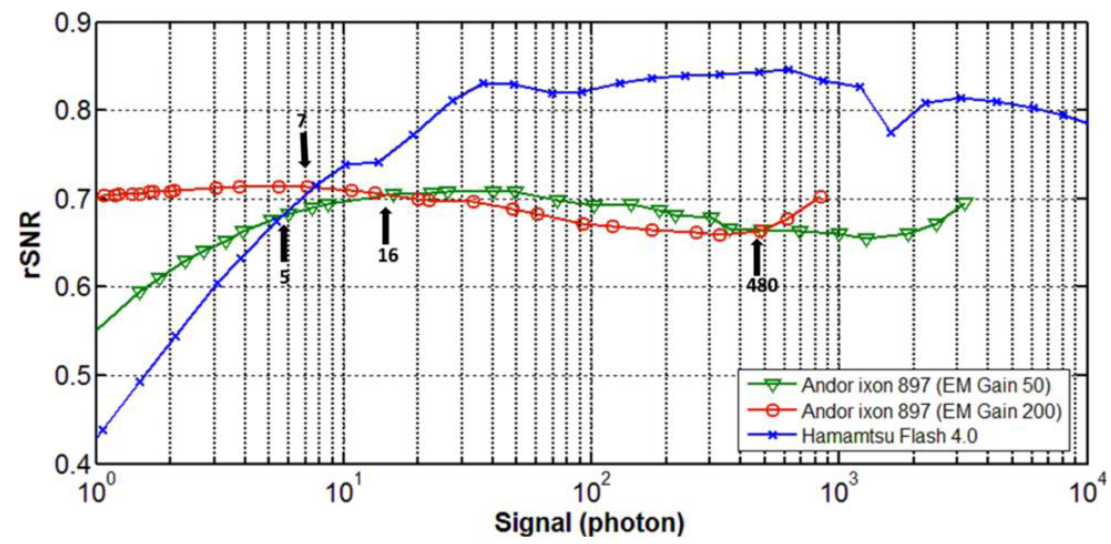

(b)

Fig. 7. Comparing the performance of a Hamamatsu Flash 4.0 sCMOS and an Andor iXon 897 EMCCD. (a) SNR curves. An enlarged view of the data inside the red rectangle is given in the right corner. (b) rSNR curves. The measurement is performed under $593 \mathrm{~nm}$, where the QE is 0.72 for the sCMOS camera and 0.96 for the EMCCD camera, respectively.

\section{Conclusion}

In this tutorial, we provide useful background information to understand the important parameters for characterizing low-light cameras, and introduce a quantitative method (called PTC) to experimentally assess the imaging performance of low-light cameras. Basing on the PTC method and its derivatives, we characterize and discuss the imaging performance of two representative low-light cameras, including a Hamamatsu Flash 4.0 CMOS camera and an Andor iXon 897 EMCCD camera. We hope this tutorial can help readers understand that the selection of a suitable camera for a specific application should be considered not only by the QE of the cameras but also different types of noise which are signal dependent.

\section{Acknowledgments}

This work was supported by National Natural Science Foundation of China (Grant Nos. 91332103, 81427801), National Basic Research Program of China (Grant No. 2015CB352003), the Program for New Century Excellent Talents in University of China (Grant No. NCET-10-0407), and the Science Fund for Creative Research Group of China (Grant No. 61421064). We thank Mr. Tadashi Maruno, Mr. Eiji Toda, Dr. Hiroyuki Kawai and Dr. Keith Bennett from Hamamatsu Photonics for the technical support and helpful discussions.

\section{References}

1. B. Moomaw, "Camera technologies for low light imaging: Overview and relative advantages," Method. Cell Biol. 114, 243-283 (2013). 
2. X. Michalet, R. A. Colyer, G. Scalia, A. Ingargiola, R. Lin, J. E. Millaud, S. Weiss, O. H. W. Siegmund, A. S. Tremsin, J. V. Vallerga, A. Cheng, M. Levi, D. Aharoni, K. Arisaka, F. Villa, F. Guerrieri, F. Panzeri, I. Rech, A. Gulinatti, F. Zappa, M. Ghioni, S. Cova, "Development of new photon-counting detectors for single-molecule fluorescence microscopy," Philosophical Trans. R. Soc. B: Biol. Sci. 368, 20120035 (2012).

3. X. Michalet, O. H. Siegmund, J. V. Vallerga, P. Jelinsky, J. E. Millaud, S. Weiss, "Detectors for single-molecule fluorescence imaging and spectroscopy," J. Mod. Opt. 54, 239 (2007).

4. L. M. Hirvonen, S. Jiggins, N. Sergent, G. Zanda, K. Suhling, "Photon counting imaging with an electron-bombarded CCD: Towards a parallelprocessing photoelectronic time-to-amplitude converter," Rev. Sci. Instrum. 85, 123102 (2014).

5. T. Brugière, F. Mayer, P. Fereyre, C. Guérin, A. Dominjon, R. Barbier, "First measurement of the inpixel electron multiplying with a standard imaging CMOS technology: Study of the EMCMOS concept," Nucl. Instrum. Methods Phys. Res. A 787, 336-339 (2015).

6. G. E. Smith, "Nobel Lecture: The invention and early history of the CCD," Rev. Mod. Phys. 82, 2307-2312 (2010).

7. R. Brewer, "The low light level potential of a CCD imaging array," IEEE. T. Electron Dev. 27, 401405 (1980).

8. H. Kröger, G. Schmidt, N. Pailer, "Faint object camera: European contribution to the hubble space telescope," Acta Astronaut. 26, 827-834 (1992).

9. M. S. Robbins, B. J. Hadwen, "The noise performance of electron multiplying charge-coupled devices," IEEE. T. Electron Dev. 50, 1227-1232 (2003).

10. E. R. Fossum, "CMOS image sensors: Electronic camera-on-a-chip," IEEE. T. Electron Dev. 44, 1689-1698 (1997).

11. M. Bigas, E. Cabruja, J. Forest, J. Salvi, "Review of CMOS image sensors," Microelectron. J. 37, 433451 (2006).

12. B. Fowler, C. Liu, S. Mims, J. Balicki, W. Li, H. Do, J. Appelbaum, P. Vu, "A 5.5 Mpixel 100 frames/sec wide dynamic range low noise CMOS image sensor for scientific applications," Proc. SPIE 7536, 753607 (2010).

13. Z. L. Huang, H. Zhu, F. Long, H. Ma, L. Qin, Y. Liu, J. Ding, Z. Zhang, Q. Luo, S. Zeng, "Localizationbased super-resolution microscopy with an sCMOS camera," Opt. Express 19, 19156-19168 (2011).

14. F. Huang, T. M. Hartwich, F. E. Rivera-Molina, Y. Lin, W. C. Duim, J. J. Long, P. D. Uchil, J. R. Myers, M. A. Baird, W. Mothes, "Video-rate nanoscopy using sCMOS camera-specific single-molecule localization algorithms," Nat. Methods 10, 653-658 (2013).
15. F. Long, S. Zeng, Z. L. Huang, "Localization-based super-resolution microscopy with an sCMOS camera Part II: Experimental methodology for comparing sCMOS with EMCCD cameras," Opt. Express 20, 17741-17759 (2012).

16. R. Yuste, A. Konnerth, Imaging in Neuroscience and Development, Cold Spring Harbor Lab. Press, New York (2004).

17. T. J. Lambert, J. C. Waters, "Assessing camera performance for quantitative microscopy," Quantitative Imaging in Cell Biology, Chap. 3, J. C. Waters, T. Wittman, Eds., pp. 35-53, Elsevier, NewYork (2013).

18. J. R. Janesick, Photon Transfer: $D N \rightarrow \lambda$, SPIE Press, Washington, USA (2007).

19. K. Narisada, D. Schreuder, Light Pollution Handbook, Springer, Netherlands (2004).

20. Labsphere, "Integrating sphere theory and applications," [Online], Available at: https://www. labsphere.com/site/assets/files/2551/a-guide-to-integrating-sphere-theory-and-applications.pdf. Accessed on 26 February 2016.

21. F. Long, S. Q. Zeng, Z. L. Huang, "Effects of fixed pattern noise on single molecule localization microscopy," Phys. Chem. Chem. Phys. 16, 21586-21594 (2014).

22. J. R. Taylor, An Introduction to Error Analysis: The Study of Uncertainties in Physical Measurements, 2nd Edition, University Science Books, Sausalito (1997).

23. E. W. Weisstein, "Standard deviation distribution." [Online], Available at: http://mathworld.wolfram. com/StandardDeviationDistribution.html.

24. E. W. Weisstein, "Sample variance distribution." [Online], Available at: http://mathworld.wolfram. com/SampleVarianceDistribution.html.

25. S. Ahn, J. A. Fessler, "Standard errors of mean, variance, and standard deviation estimators", [Online], Available at: https://pdfs.semanticscholar. org/ba2b/131bc7b442c3f7f4641339f3549f69b15a9b. pdf. [Accessed: 26-Feb-2016].

26. Andor, "Sensitivity", [Online], Available at: http:// www.andor.com/learning-academy/sensitivity-making-sense-of-sensitivity. Accessed on 26 February 2016.

27. H. Tian, Noise analysis in CMOS image sensors, Ph.D. Thesis, Stanford University, CA, (2000).

28. PCO, "pco sCMOS brochure", [Online], Available at: http://www.pco.de/fileadmin/user_upload/pcoproduct_sheets/pco_sCMOS_brochure.pdf. Accessed on 26 February 2016.

29. Hamamatsu, "ORCA-Flash4.0 V2", [Online], Available at: https://www.hamamatsu.com/resources/ pdf/sys/SCAS0081E_C11440-22CU.pdf. Accessed on 26 February 2016. 